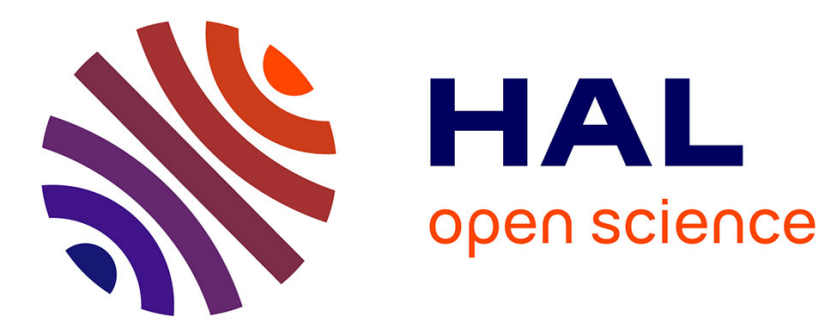

\title{
Limited coagulation-diffusion dynamics in inflating spaces
}

\author{
Jean-Yves Fortin, Xavier Durang, Mooyoung Choi
}

\section{To cite this version:}

Jean-Yves Fortin, Xavier Durang, Mooyoung Choi. Limited coagulation-diffusion dynamics in inflating spaces. The European Physical Journal B: Condensed Matter and Complex Systems, 2020, 93, pp.175. 10.1140/epjb/e2020-10058-9 . hal-02913488v2

\section{HAL Id: hal-02913488 \\ https://hal.science/hal-02913488v2}

Submitted on 20 Aug 2020

HAL is a multi-disciplinary open access archive for the deposit and dissemination of scientific research documents, whether they are published or not. The documents may come from teaching and research institutions in France or abroad, or from public or private research centers.
L'archive ouverte pluridisciplinaire HAL, est destinée au dépôt et à la diffusion de documents scientifiques de niveau recherche, publiés ou non, émanant des établissements d'enseignement et de recherche français ou étrangers, des laboratoires publics ou privés. 


\title{
Limited coagulation-diffusion dynamics in inflating spaces
}

\author{
Jean-Yves Fortin ${ }^{1,3}$, Xavier Durang ${ }^{2}$, and MooYoung Choi ${ }^{1}$ \\ ${ }^{1}$ Department of Physics and Astronomy and Center for Theoretical Physics, Seoul \\ National University, Seoul 08826, Korea \\ 2 Department of Physics, University of Seoul, Seoul 02504, Korea \\ ${ }^{3}$ Laboratoire de Physique et Chimie Théoriques, CNRS (UMR 7019), Université de \\ Lorraine, F54000 Nancy, France \\ E-mail: jean-yves.fortin@univ-lorraine.fr,mychoi@snu.ac.kr
}

\begin{abstract}
We consider the one-dimensional coagulation-diffusion problem on a dynamical expanding linear lattice, in which the effect of the coagulation process is balanced by the dilatation of the distance between the particles. Distances $x(t)$ follow the general law $\dot{x}(t) / x(t)=\alpha(1+\alpha t / \beta)^{-1}$ with growth rate $\alpha$ and exponent $\beta$, describing both algebraic and exponential $(\beta=\infty)$ growths. In the space continuous limit, the particle dynamics is known to be subdiffusive, with the diffusive length varying like $t^{1 / 2-\beta}$ for $\beta<1 / 2$, logarithmic for $\beta=1 / 2$, and reaching a finite value for all $\beta>1 / 2$. We interpret and characterize quantitatively this phenomena as a second order phase transition between an absorbing state and a localized state where particles are not reactive. We furthermore investigate the case when space is discrete and use a generating function method to solve the time differential equation associated with the survival probability. This model is then compared with models of growth on geometrically constrained two-dimensional domains, and with the theory of fractional diffusion in the subdiffusive case. We found in particular a duality relation between the diffusive lengths in the inflating space and the fractional theory.
\end{abstract}

PACS numbers: $05.10 . \mathrm{Gg}, 05.40 .-\mathrm{a}, 05.40 . \mathrm{Fb}$

\section{Introduction}

Cooperative effects in low-dimensional systems with strongly interacting particles present a rich variety of critical properties in the regime out of equilibrium. Model examples of such systems are provided by one dimensional reaction-diffusion processes $[1$, $2,3]$, which are relevant to a number of non-equilibrium physical cases, such as excitons in polymer chains $\mathrm{TMMC}=\left(\mathrm{CH}_{3}\right)_{4} \mathrm{~N}\left(\mathrm{MnCl}_{3}\right)$ [4] or relaxation of photoexcitations in carbon-nanotubes [5]. The low dimensionality induces strong fluctuations that dominate the kinetics, and invalidates all descriptions based on mean-field theories below the upper critical dimension $d_{c}=2$. Exact results are therefore of importance in describing the dynamical behavior with precision by taking into account all the correlations. 
Here we consider one of these coagulation-diffusion processes on a one-dimensional lattice with discrete sites of elementary size $a$. The Markov dynamics is defined by the particle elementary moves at the same rate $\tau^{-1}: A+\emptyset \rightarrow \emptyset+A$ or $\emptyset+A \rightarrow A+\emptyset$ for the diffusion process, and $A+A \rightarrow A+\emptyset$ or $A+A \rightarrow \emptyset+A$ for the irreversible coalescence process with probability one. It is known that this out of equilibrium dynamics tends to decrease the concentration of particles with time like $t^{-1 / 2}[6,1]$, instead of $t^{-1}$ in the mean field description, hence driving the system to an absorbing state consisting of one particle.

External sources such as particle input $[7,8]$ leads on the contrary to an equilibrium state, and the model can be solved using for example the eigenvalue formalism. The opposite case of a source of particles would be insertion of empty spaces, which can be modeled by expanding the space or the geometry uniformly and independently of the presence of particles, modifying the Brownian dynamics and therefore the Fokker-Planck equation $[9,10,11]$. This has many practical applications for example in the physics of bubble coalescence in expanding foams $[12,13]$, or gravitational merging of galaxies or masses as well as ray diffusion in an inflating universe [14]. Expanding media also have a strong influence on the dynamics of cells induced by tissue growth in biology $[15,16,17]$. In particular, geometrical constraints in two-dimensional competitive environments with gene segregation for two different kinds of bacteria [18, 19], such as Eschrichia coli, exert also an influence on the growth of the different colonies when it is directed radially in presence of decimation at the boundaries between two different colonies $[18,15]$. In this radial configuration, the frontiers or domain boundaries between two competitive colonies display superdiffusive spatial fluctuations with a wandering exponent $0.65>1 / 2$, greater than the exponent of the simple Brownian diffusion.

In this paper we investigate the effect of geometrical time expansion for Brownian particles with coalescence. We review in section 2 the general results about the diffusion properties of a single particle using a discrete analysis (discrete space and time), and in the continuous limit identifying two different regimes corresponding to subdiffusion and localization.

Section 3 is dedicated to describing analytically the dynamics of many particles with coalescence through diffusion, using the results of the previous section obtained in the literature and the empty interval method in the continuous case. We identify the two regimes as a second order phase transition, using the number of particles as the order parameter and the expansion exponent as the inverse of a temperature. In particular, we can show the model presents slowing down characteristics at the transition.

In section 4, we develop an algorithm for the stochastic process in an expanding medium, using a combination of continuous and discrete methods for the space and particle dynamics respectively. The results confirm the analytical and scaling expressions for the particle concentration of section 3 in the different regimes.

In section 5 , we extend the solution in the case where space is discrete and time continuous. We propose two methods to solve the corresponding diffusion equation for the empty interval distribution relevant for various physical systems, and compare the 
solutions with the numerical results of section 4 .

In section 6, we discuss the relevance and connection of the model with the problem of particle diffusion in constrained two-dimensional geometries, including radial constraints and generalized Brownian noise.

Finally, in section 7, we consider the model in the more general context of abnormal diffusion described by the action of fractional operators. The aim is to examine the correspondence and analogy between the problem of diffusion in expanding space and the formalism of diffusion with adequate fractional operators, involving continuous time random walk (CTRW) models. We shall conclude from this analysis of the differences and relevance of the fractional formalism. We establish in particular a duality relation between diffusive lengths which holds for all the regimes.

\section{Effective diffusion length}

To define more precisely the problem, we consider a lattice initially filled randomly with particles at a given concentration $c_{0}$, and we constraint the lengths between any two spatial points of the lattice to increase with time at a certain rate (or by adding for example empty sites on a discrete chain uniformly at random locations), with distances growing with time like a power or exponential law. The space inflation tends to increase distances between the particles, preventing them to coagulate by diffusive motion. It is expected that the two opposite contributions (diffusive motion and space inflation) will induce a transition between two regimes. To analyze in detail this phenomenon, let assume that all distances $x(t)$ increase according to the general law

$$
\frac{x\left(t^{\prime}\right)}{x(t)}=\left(\frac{1+\alpha t^{\prime} / \beta}{1+\alpha t / \beta}\right)^{\beta}
$$

where $\beta$ is a given exponent and $\alpha$ is the growth rate of the lattice, or the amount of new empty spaces added uniformly to the lattice per unit of time. The dynamics of the lattice modifies the diffusive length of particles. Indeed, we can evaluate the diffusive length in this system by considering the stochastic motion of a single particle: If we define the growth factor $r(t)=(1+\alpha t / \beta)^{\beta}$, and $x_{k}$ the position of the particle at time $t_{k}=k \tau$, where $\tau$ is the unit of time, we can write the discrete equation of motion as

$$
x_{k}=\frac{r_{k}}{r_{k-1}}\left(x_{k-1}+\xi_{k}\right)
$$

where $x_{0}=0, r_{k}=r\left(t_{k}\right)$, and $\xi_{k}$ a Gaussian white noise satisfying $\left\langle\xi_{k}\right\rangle=0$ and $\left\langle\xi_{k} \xi_{k^{\prime}}\right\rangle=2 a^{2} \delta_{k, k^{\prime}}$. Solving the previous equation leads directly to

$$
x_{k}=\frac{r_{k}}{r_{k-1}} \xi_{k}+\frac{r_{k}}{r_{k-2}} \xi_{k-2}+\cdots+\frac{r_{k}}{r_{0}} \xi_{1} .
$$

The typical mean squared displacement of the random walk $\left\langle x_{k}^{2}\right\rangle$ is found to be equal to

$$
\begin{aligned}
\left\langle x_{k}^{2}\right\rangle & =2 a^{2} r_{k}^{2}\left[1+\frac{1}{r_{1}^{2}}+\cdots+\frac{1}{r_{k-1}^{2}}\right] \\
& =2 a^{2} r_{k}^{2} \sum_{j=0}^{k-1} \frac{1}{(1+\alpha \tau j / \beta)^{2 \beta}}
\end{aligned}
$$


from which we can extract an effective or comoving diffusive length $\ell\left(t_{k}\right)=\left\langle x_{k}^{2}\right\rangle^{1 / 2} / r_{k}$ whose long time behavior depends on the value of $\beta$. We first define the continuum limit $a \rightarrow 0$ and $\tau \rightarrow 0$, such that the diffusive constant $\mathscr{D}=a^{2} / \tau$ remains finite, which gives [11]

$$
\ell^{2}(t)=2 \mathscr{D} \frac{\beta}{\alpha(1-2 \beta)}\left[(1+\alpha t / \beta)^{1-2 \beta}-1\right] .
$$

If $0 \leq \beta<1 / 2$, we have $\ell(t) \sim t^{1 / 2-\beta}$, the effective diffusive length increases but less than the usual $\ell(t)=\sqrt{2 \mathscr{D}} t^{1 / 2}$ Brownian motion length and the process is subdiffusive $[20,21,22]$. If $\beta=1 / 2$, the length diverges logarithmically $\ell^{2}(t) \sim(\mathscr{D} / \alpha) \log (1+2 \alpha t)$, and if $\beta>1 / 2$, it reaches a finite value $\ell^{2}(t) \simeq 2 \mathscr{D} \beta / \alpha(2 \beta-1) \ddagger$. In particular, in the case of an exponential growth, $\beta=\infty, \ell^{2}(t)=\mathscr{D}\left(1-e^{-2 \alpha t}\right) / \alpha \simeq \mathscr{D} / \alpha$. When $\beta=0$ and/or $\alpha=0$, we recover the usual Brownian law $\ell(t) \sim t^{1 / 2}$. We therefore expect from this analysis that the coagulation-reaction process will stop when $\beta>1 / 2$ after a transit time since effectively the motion of the particles will be frozen. This limit is similar to a dynamical localization of the particle, as its motion is impeded by the space expansion.

\section{Empty interval distribution method for the particle concentration}

In order to find the time dependence of the particle concentration, we need to solve the reaction-diffusion problem. It is convenient to use the empty interval method based on the interparticle distribution function (IPDF) [23], with which the problem becomes linear and solvable: We define the probability to have at least $n$ consecutive empty spaces $\circ$ by

$$
E(n, t)=\operatorname{Prob}(\times \underbrace{o \cdots \circ}_{n} \times)
$$

where $\times$ represents equivalently either a particle $\bullet$ or an empty space $\circ$. As before, we assume that $\tau^{-1}$ is the rate of diffusion to the right or left at a given time step. By considering the gains and losses of particles at the boundaries of an interval of size $n(t)$, one can express the rate of change for $E(n(t), t)$ with respect with the expanding interval $n(t)$ by writing the total derivative

$$
\begin{aligned}
\frac{d E(n(t), t)}{d t} & =\frac{\partial E(n(t), t)}{\partial t}+\dot{n}(t) \frac{\partial E(n(t), t)}{\partial n(t)} \\
& =\frac{2}{\tau} \Delta E(n(t), t)
\end{aligned}
$$

with $\dot{n}(t)=\alpha n(t)(1+\alpha t / \beta)^{-1}$, and $\Delta$ is the discrete Laplacian operator: $\Delta E(n)=$ $E(n+1)+E(n-1)-2 E(n)$. The interval distribution $E(n, t)$ is therefore determined by the partial differential equation

$$
\frac{\partial E(n, t)}{\partial t}=\frac{2}{\tau} \Delta E(n, t)-\frac{\alpha}{1+\alpha t / \beta} n \frac{\partial E(n, t)}{\partial n} .
$$

$\ddagger$ In reference [11], the corresponding parameters are the following: $\ell^{2}(t) \rightarrow 2 D \tau(t), \beta \rightarrow \gamma, \beta / \alpha \rightarrow t_{0}$ and $\alpha \rightarrow H$ 
The operator $n \partial_{n}$ is the usual dilatation operator and appears naturally in this equation. To solve equation (8), we consider the continuum limit of the space variable as follows. The discrete case will be investigated in section 5 . In the limit $a \rightarrow 0$, we introduce continuous space coordinates $x=n a$ and diffusion coefficient $\mathscr{D}=a^{2} / \tau$. The time evolution of the IPDF then becomes

$$
\frac{\partial E(x, t)}{\partial t}=2 \mathscr{D} \frac{\partial^{2} E(x, t)}{\partial x^{2}}-\frac{\alpha x}{1+\alpha t / \beta} \frac{\partial E(x, t)}{\partial x} .
$$

It is convenient to perform the transformations $y=x r^{-1}(t)$ as the new comoving space variable, and $u=u(t)$ as new time variable. This change of variables simplifies the previous equation which becomes

$$
\frac{\partial \mathcal{E}(y, u)}{\partial u}=2 \mathscr{D} \frac{\partial^{2} \mathcal{E}(y, u)}{\partial^{2} y}
$$

where $\mathcal{E}(y, u)=E(x, t)$, provided that $u(t)$ satisfies

$$
\begin{aligned}
& \dot{u}(t)=r^{-2}(t), u(0)=0 \\
& u(t)=\frac{\beta}{\alpha(1-2 \beta)}\left[(1+\alpha t / \beta)^{1-2 \beta}-1\right]=\frac{\ell^{2}(t)}{2 \mathscr{D}} .
\end{aligned}
$$

We can therefore relate naturally the proper time $u(t)$ to the effective diffusive length defined in equation (5). Equation (10) is the usual diffusion equation. The Laplace transform $\hat{\mathcal{E}}(y, s)$ with respect to the time variable $u$ verifies the differential equation

$$
s \hat{\mathcal{E}}(y, s)-E(y, 0)=2 \mathscr{D} \frac{\partial^{2} \hat{\mathcal{E}}(y, s)}{\partial^{2} y}
$$

where $\mathcal{E}(y, 0)=E(y, 0)$ is the initial condition for the interval distribution, with $y=x$ at $t=0$. We will consider in the following an initial exponential distribution $E(y=x, 0)=e^{-c_{0} x}$, for which the average particle density is $c_{0}$. The solution of this differential equation is simply given by two exponential functions, in addition to the particular solution associated to $E(y, 0)$

$$
\begin{aligned}
& \hat{\mathcal{E}}(y, s)=a(s) \exp \left(-\sqrt{\frac{s}{2 \mathscr{D}}} y\right)+b(s) \exp \left(\sqrt{\frac{s}{2 \mathscr{D}}} y\right) \\
& +\frac{1}{\sqrt{8 \mathscr{D} s}}\left[\exp \left(-\sqrt{\frac{s}{2 \mathscr{D}}} y\right) \int_{0}^{y} d y^{\prime} \exp \left(\sqrt{\frac{s}{2 \mathscr{D}}} y^{\prime}\right) E\left(y^{\prime}, 0\right)\right. \\
& \left.-\exp \left(\sqrt{\frac{s}{2 \mathscr{D}}} y\right) \int_{0}^{y} d y^{\prime} \exp \left(-\sqrt{\frac{s}{2 \mathscr{D}}} y^{\prime}\right) E\left(y^{\prime}, 0\right)\right] .
\end{aligned}
$$

Coefficients $a(s)$ and $b(s)$ depend only on $s$. They are determined by the two conditions:

$$
\mathcal{E}(0, u)=1, \lim _{y \rightarrow \infty} \mathcal{E}(y, u)=0 .
$$

The first one implies $\hat{\mathcal{E}}(0, s)=1 / s$, and fixes the value of $a(s)+b(s)=1 / s$. Since $\hat{\mathcal{E}}(y, s)$ should remain finite when $y \rightarrow \infty$, the second condition restricts $b(s)$ to be equal to

$$
b(s)=\frac{1}{\sqrt{8 \mathscr{D} s}} \int_{0}^{\infty} d y^{\prime} \exp \left(-\sqrt{\frac{s}{2 \mathscr{D}}} y^{\prime}\right) E\left(y^{\prime}, 0\right) .
$$


We then use the following inverse Laplace functions when $x>0[24]$

$$
\begin{aligned}
& \mathcal{L}^{-1}\left(\frac{1}{s} \exp \left(-\sqrt{\frac{s}{2 \mathscr{D}} y}\right)\right)=\operatorname{erfc}\left(\frac{y}{\sqrt{8 \mathscr{D} u}}\right) \\
& \mathcal{L}^{-1}\left(\frac{1}{\sqrt{s}} \exp \left(-\sqrt{\frac{s}{2 \mathscr{D}} y}\right)\right)=\frac{1}{\sqrt{\pi u}} \exp \left(-\frac{y^{2}}{8 \mathscr{D} u}\right)
\end{aligned}
$$

to obtain the general expression of $\mathcal{E}(y, u)$

$$
\begin{aligned}
\mathcal{E}(y, u) & =\operatorname{erfc}\left(\frac{y}{\sqrt{8 \mathscr{D} u}}\right)+\frac{1}{\sqrt{8 \mathscr{D} \pi u}} \int_{0}^{\infty} d y^{\prime}\left[\exp \left(-\frac{\left(y-y^{\prime}\right)^{2}}{8 \mathscr{D} u}\right)\right. \\
& \left.-\exp \left(-\frac{\left(y+y^{\prime}\right)^{2}}{8 \mathscr{D} u}\right)\right] E\left(y^{\prime}, 0\right) .
\end{aligned}
$$

The particle concentration is determined by the relation $c(t)=-\left.\partial_{x} E(x, t)\right|_{x=0}=$ $-\left.r^{-1}(t) \partial_{y} \mathcal{E}(y, u)\right|_{y=0}$, and the number of particles as function of time for a system initially of size $L_{0}$ is then equal to $N_{p}(t)=c(t) L_{0} r(t)=-\left.L_{0} \partial_{y} \mathcal{E}(y, u)\right|_{y=0}$. If the initial number of particles is $N_{0}=c_{0} L_{0}$, then the ratio $N_{p}(t) / N_{0}$ is equal to, for an initial exponential distribution of empty intervals, see also [11]

$$
\begin{aligned}
\frac{N_{p}(t)}{N_{0}} & =e^{c_{0}^{2} 2 \mathscr{D} u(t)} \operatorname{erfc}\left(c_{0} \sqrt{2 \mathscr{D} u(t)}\right) \\
& =e^{c_{0}^{2} \ell^{2}(t)} \operatorname{erfc}\left(c_{0} \ell(t)\right)
\end{aligned}
$$

where $\ell(t)$ is the effective diffusive length given by equation (11). When $\beta<1 / 2$, $\ell(t) \simeq t^{1 / 2-\beta}$ and the number of particle decreases like a power law $N_{p}(t) / N_{0} \simeq$ $\left(\sqrt{\pi} c_{0} \ell(t)\right)^{-1} \sim t^{-\nu}$, with exponent $\nu=1 / 2-\beta$. When $\beta=1 / 2$, the number of particle decreases logarithmically like $\log (t)^{-1 / 2}$.

In the case $\beta>1 / 2$, the number of particles, after a regime of coalescence where it decreases, reaches a finite limit given by the dimensionless parameter $\lambda$ defined in the following

$$
\begin{aligned}
\frac{N_{p}(\infty)}{N_{0}} & =e^{\lambda^{2}} \operatorname{erfc}(\lambda), \lambda=\sqrt{\frac{2 \beta \mathscr{D}}{\alpha(2 \beta-1)}} c_{0} \\
& \simeq \frac{1}{\sqrt{\pi} \lambda}\left(1-\frac{1}{2 \lambda^{2}}\right), \lambda \gg 1 \\
& \simeq 1-\frac{2 \lambda}{\sqrt{\pi}}, \lambda \ll 1 .
\end{aligned}
$$

For short times, $\alpha t \ll 1$, the coagulation process is not affected by the space expansion since $\ell(t) \simeq \sqrt{2 \mathscr{D} t}$. The result in equation (18) is similar to the concentration of the purely diffusive-coalescence model $(\alpha=0)$ as function of time [25]

$$
c(t)=c_{0} e^{c_{0}^{2} \ell_{0}^{2}(t)} \operatorname{erfc}\left(c_{0} \ell_{0}(t)\right) \simeq(2 \pi \mathscr{D} t)^{-1 / 2}
$$

where $\ell_{0}(t)=\sqrt{2 \mathscr{D} t}$ replaces $\ell(t)$ instead. Identifying this expression with equation (18), we can evidence the crossover regime of dissociation at which the coagulation process due to the diffusive motion stops at a characteristic time defined by $\ell_{0}(t) c_{0}=\lambda$, and which is equal to $t_{c}=\beta /[\alpha(2 \beta-1)]$. 
It is interesting to make an analogy with phase transition phenomena. Indeed we can consider $N_{p} / N_{0}$ as an order parameter and $\beta$ as the inverse of a temperature, with $\beta_{c}=1 / 2$ the critical point. In the steady state limit, the previous results can be summarized as follows:

$$
\begin{aligned}
& \frac{N_{p}(\infty)}{N_{0}}=0, \beta<\beta_{c} \\
& \frac{N_{p}(\infty)}{N_{0}} \simeq \sqrt{\frac{\alpha}{\pi \mathscr{D} c_{0}^{2}}} \sqrt{1-\beta_{c} / \beta}, \beta \gtrsim \beta_{c} .
\end{aligned}
$$

This corresponds, by identification, to a second order phase transition, between an absorbing state (high temperature) and a localized state of particles (low temperature) where $N_{p}$ is the number of localized particles. In terms of electronic localization, the critical point may describe the transition between fully delocalized electrons and partially localized electrons, $\beta$ being a parameter similar to the strength of a random potential on a one-dimensional lattice, or inverse temperature. The critical slowing down phenomenon at $\beta=\beta_{c}$ is evidenced by the fact that the effective diffusive length is diverging logarithmically. We can also notice that the remaining number of particles in inversely proportional to the concentration since the coagulation reactions are more effective when more particles are initially closer to each other, before the dissociation time occurs.

\section{Numerical simulations}

The analytical result given by equation (18) is compared to numerical simulations, first in the subdiffusive case $\beta \leq 1 / 2$, see figures 1 and $2 . N_{0}$ particles are randomly distributed on a chain of size $L_{0}$ with an intrinsic unit step size $\Delta x$. At each time step $\Delta t$, a site of width $\Delta x$ is randomly chosen. If it is occupied by a particle, this one diffuses by one step $\Delta x$ on the right or left with equal probability. If the destination site contains another particle within the distance $\Delta x$, the two particles coalesce. After this process, the size of the system, as well as the distances between the remaining particles, are increased by a factor $r(t+\Delta t) / r(t)$. The number of particles is then evaluated after $10^{7}$ time steps by averaging over a set of random initial samples. The evolution of the number of particles is displayed in figure 1 for an initial system size $L_{0}=1000$ and $c_{0}=N_{0} / L_{0}=1$. In the inset, the approximate power law exponent $\nu$ is evaluated and compared to the exact expression $\nu=1 / 2-\beta$. For comparison, in figure 2 is plotted the function given by equation (18) for several values of $\beta \leq 1 / 2$.

In the exponential case $(\beta=\infty)$, we have plotted in figure 3 the numerical value of the ratio of particles for a linear chain of $L_{0}=1000$ sites and different values of $\alpha$, as function of the dimensionless time variable $\alpha t$, and for an initial concentration $c_{0}=1$. In inset is displayed the final value of the remaining number of particles, as function of $\lambda=c_{0}(\mathscr{D} / \alpha)^{1 / 2}$, with the diffusive constant we have estimated to be equal to $\mathscr{D} \simeq\left(8 N_{0}\right)^{-1 / 2}$ in dimensionless units. This gives a good agreement with the numerical data. The analytical solution, equation (19), is represented in figure 4 for different values 


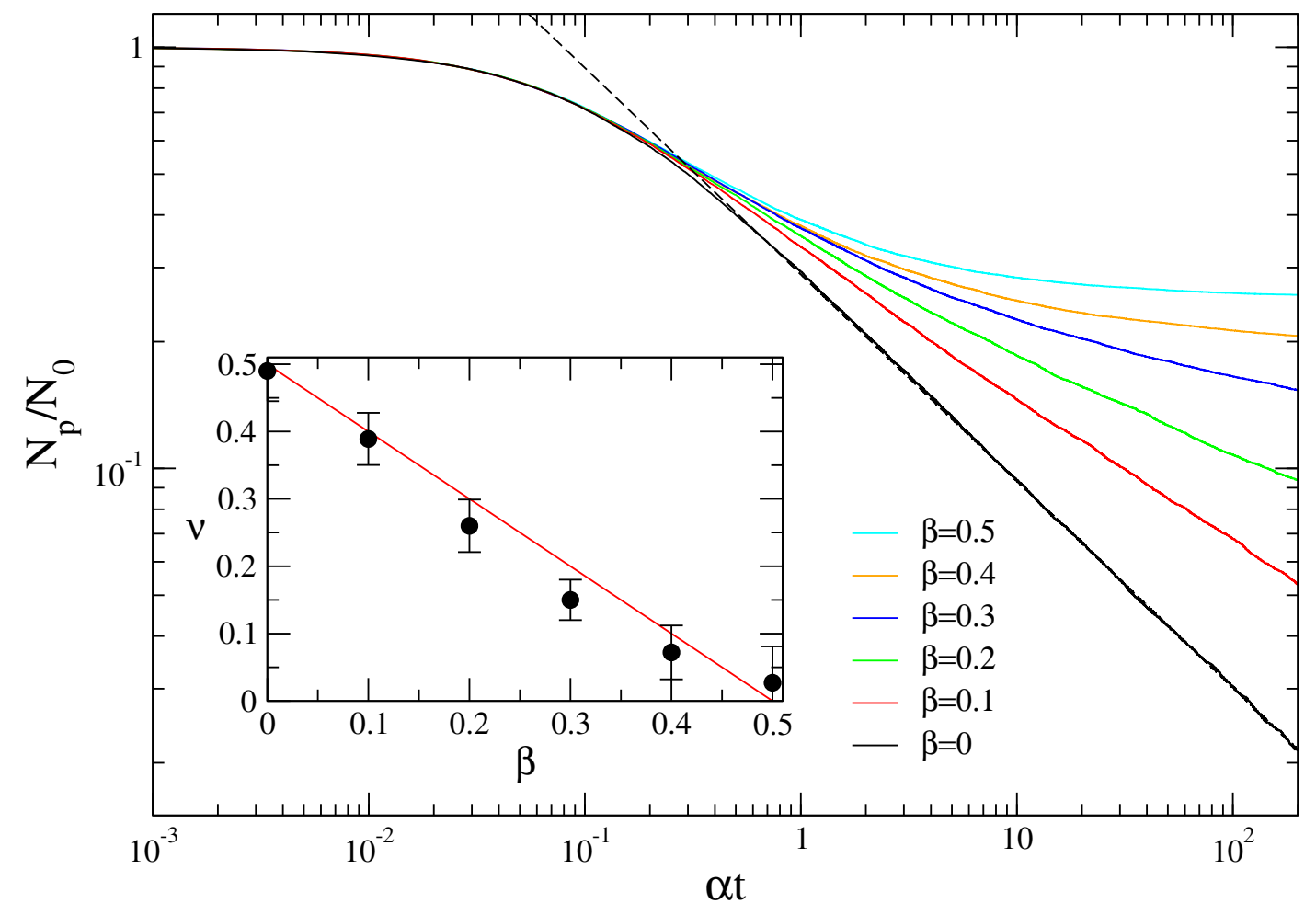

Figure 1. Numerical evaluation of the ratio of remaining number of particles $N_{p} / N_{0}$ for several values of $\beta$. Initial parameters are $N_{0}=L_{0}=1000$ and $\alpha=10^{-4}$. The dashed line represents the asymptotic power law $t^{-\nu}=t^{-1 / 2}$ when $\beta=0$. Inset: Decay exponent $\nu$ extracted from the asymptotic behavior as function of $\beta$. Red line is the expected theoretical result: $\nu=1 / 2-\beta$.

of $\lambda$ and $\alpha=10^{-4}$. The red dashed curve represents the time evolution of the particle number in absence of expansion, which shows approximately where the dissociation time $t_{c}$ is located.

\section{Discrete solution for the IPDF}

In this section, we investigate the solutions of the linear partial differential equation (8), when the time is continuous but space discrete, using generating functions. Mathematically, similar discrete equations have been solved using Poisson-Jacobi transform, see for example reference [26], but only in the case where the coefficients are independent of time. We start by writing equation (8) in a symmetric form

$$
\begin{aligned}
\tau \frac{\partial E(n, t)}{\partial t} & =2 E(n+1, t)-4 E(n, t)+2 E(n-1, t) \\
& -\frac{\alpha \tau / 2}{1+\alpha t / \beta} n(E(n+1, t)-E(n-1, t))
\end{aligned}
$$

with constraint $E(0, t)=1$. We first notice that extending the interval length $n$ to negative values is possible using the symmetry $E(-n, t)=2-E(n, t)$, see reference [25]. This relation can be found easily in the pure diffusive case when $\alpha=0$ but also 


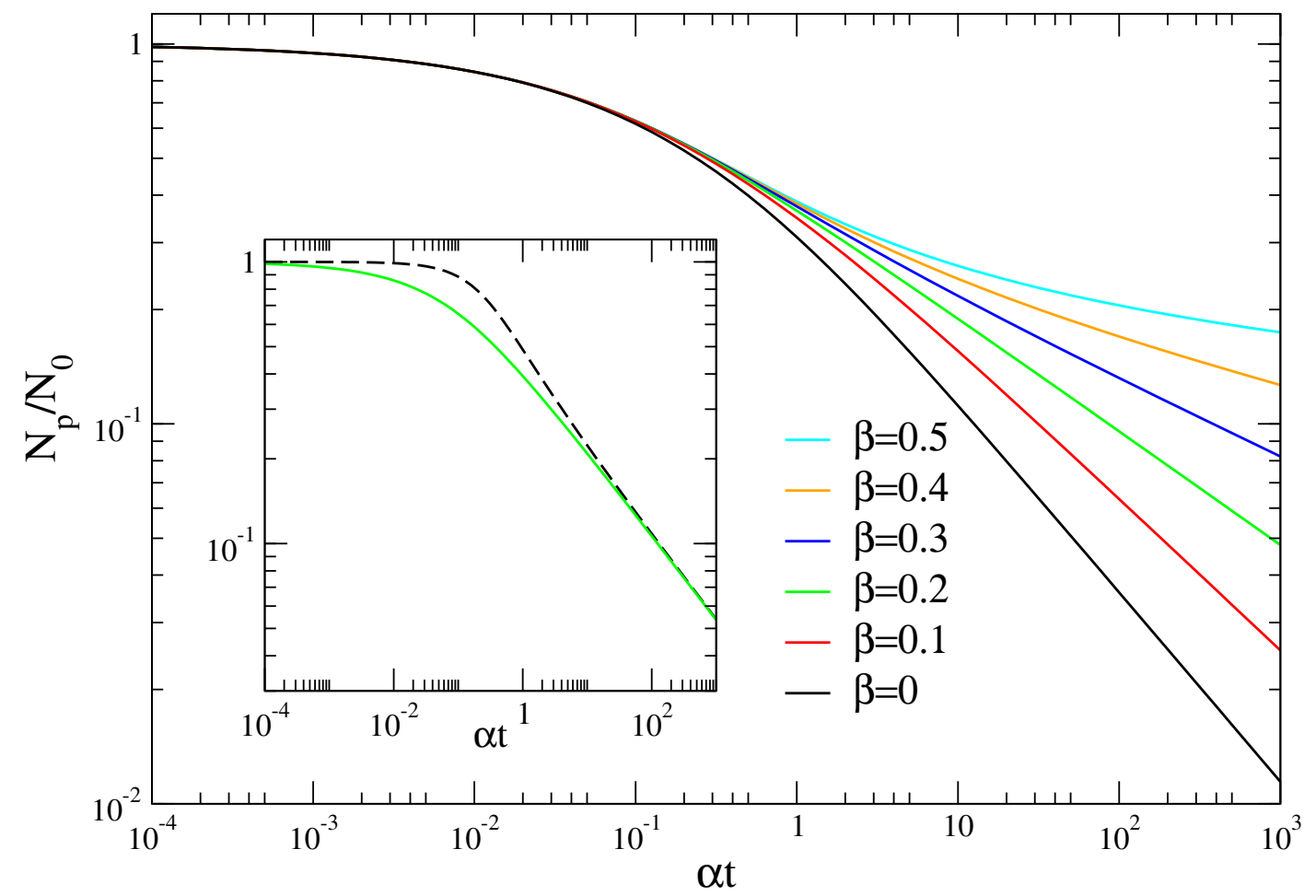

Figure 2. Analytical evaluation of the remaining number of particles from equation (18), for several values of $\beta$. Parameters are chosen such that $\mathscr{D}=1 /\left(8 N_{0}\right), c_{0}=1$ and $\alpha=10^{-4}$ in dimensionless units. Inset: Comparison between expression (18) (green line) and the discrete solution (dashed line), see equation (44), for $\beta=0.2$ and $\alpha \tau=1$.

holds here for equation (22) and can be proved by recurrence. To solve the case $\alpha \neq 0$, we consider the generating function $G(z, t)=\sum_{n} E(n, t) z^{n}$. The differential equation satisfied by $G(z, t)$ is therefore

$$
\begin{aligned}
& \tau \frac{\partial G(z, t)}{\partial t}=\left[(2+\gamma(t))\left(z+\frac{1}{z}\right)-4\right] G(z, t)-\gamma(t)\left(1-z^{2}\right) \frac{\partial G(z, t)}{\partial z}, \\
& \gamma(t)=\frac{\alpha \tau / 2}{1+\alpha t / \beta}=\frac{\tau}{2} \frac{\dot{r}(t)}{r(t)}
\end{aligned}
$$

It is important to note that this equation is invariant by transformation $z \rightarrow 1 / z$. A convenient way to reduce the previous equation is to use the change of variable $y=r(t)(1-z) /(1+z)$, for which the new function $\tilde{G}(y, t)=G(z, t)$ satisfies

$$
\tau \frac{\partial \tilde{G}(y, t)}{\partial t}=\left[(2+\gamma(t))\left(\frac{r(t)-y}{r(t)+y}+\frac{r(t)+y}{r(t)-y}\right)-4\right] \tilde{G}(y, t)
$$

the solution of which is

$$
\tilde{G}(y, t)=F(y) \exp \left(-\frac{4 t}{\tau}+\int_{0}^{t} \frac{d t^{\prime}}{\tau}\left(2+\gamma\left(t^{\prime}\right)\right)\left(\frac{r\left(t^{\prime}\right)-y}{r\left(t^{\prime}\right)+y}+\frac{r\left(t^{\prime}\right)+y}{r\left(t^{\prime}\right)-y}\right)\right) .
$$

Therefore, we obtain after some algebra and using the variable $w=(1-z) /(1+z)$

$$
G(z, t)=r(t) F(w r(t)) \exp \left(4 \int_{0}^{t} \frac{d t^{\prime}}{\tau}\left(2+\gamma\left(t^{\prime}\right)\right) \sum_{k \geq 1}^{\infty} w^{2 k} \frac{r^{2 k}(t)}{r^{2 k}\left(t^{\prime}\right)}\right)
$$




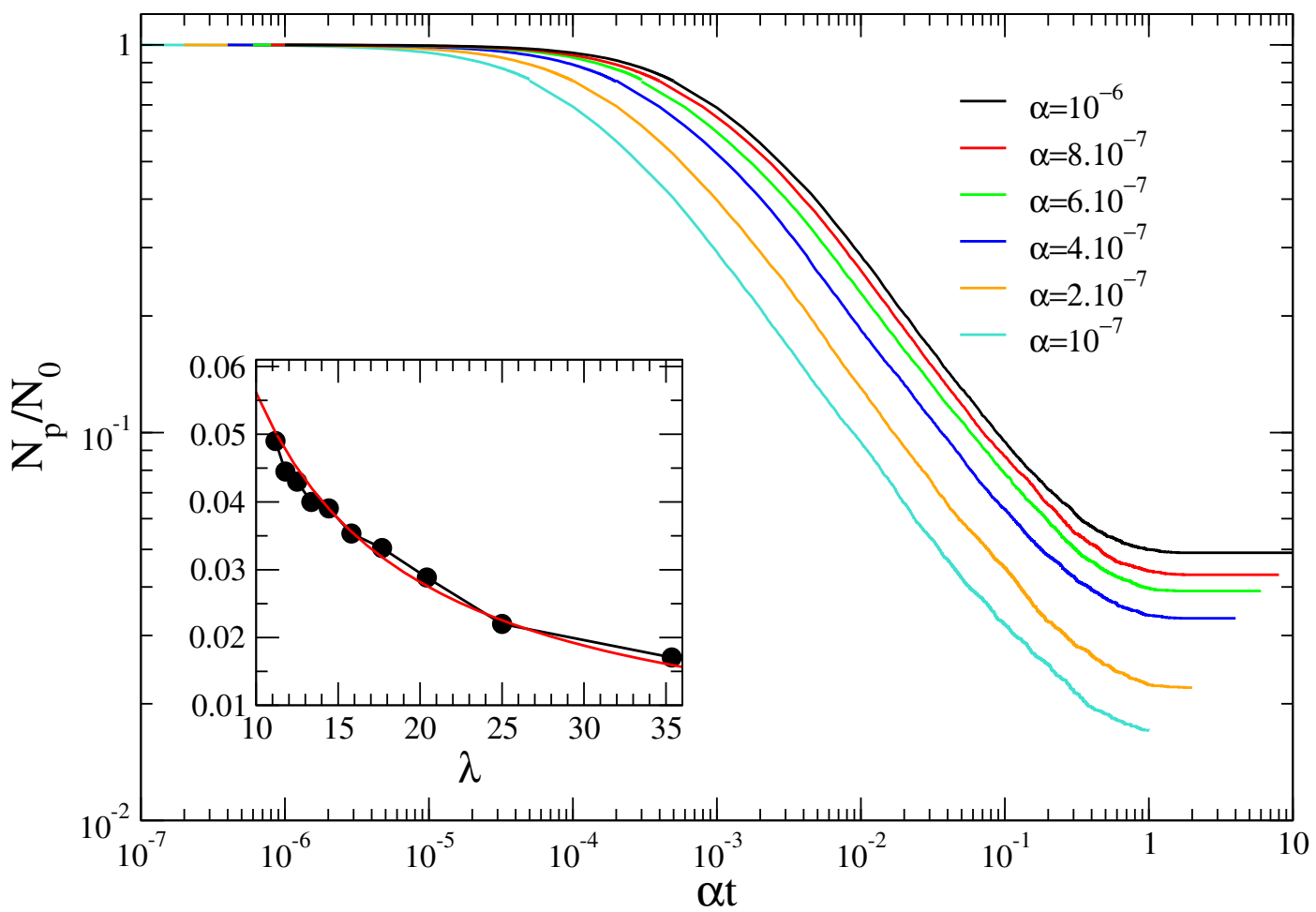

Figure 3. Numerical evaluation of the ratio $N_{p} / N_{0}$ in the exponential case, for several expansion rates $\alpha$. Initial parameters are $N_{0}=L_{0}=1000$. Inset: Number of remaining particles as function of $\lambda$. The red curve is the fit using equation (19) with $\lambda=\left(8 N_{0} \alpha\right)^{-1 / 2}$.

$$
=F(w r(t)) \frac{\left(1-w^{2}\right) r(t)}{1-w^{2} r^{2}(t)} \exp \left(\frac{8 \beta}{\alpha \tau} \sum_{k=1}^{\infty} \frac{w^{2 k}}{2 \beta k-1}\left[r^{2 k}(t)-1-\frac{\alpha t}{\beta}\right]\right) .
$$

The sum over $k$ is related in the general case for $\beta$ to Lerch transcendent function

$$
\sum_{k \geq 1} \frac{z^{k}}{2 \beta k-1}=\frac{z}{2 \beta} \Phi\left(z, 1,1-\frac{1}{2 \beta}\right) .
$$

In the limit $\beta=0$ or $r(t)=1$, after approximating the factor $r^{2 k}(t) \simeq 1+2 k \alpha t$, we recover the generating function for the diffusive case in terms of modified Bessel functions

$$
\begin{aligned}
G(z, t) & =F(w) \exp \left(-\frac{4 t}{\tau}+2\left(z+\frac{1}{z}\right) \frac{t}{\tau}\right) \\
& =G(z, 0) \exp \left(-\frac{4 t}{\tau}\right) \sum_{n} I_{n}\left(\frac{4 t}{\tau}\right) z^{n}
\end{aligned}
$$

since $F(w)=G(z, 0)=\sum_{m} E(m, 0) z^{m}$. We obtain, by identification of the power $z^{n}$ in equation (27) and using the symmetry relation $E(-n, t)=2-E(n, t)$, the following expansion, generalizing previous results [27]

$$
E(n, t)=e^{-4 t / \tau} \sum_{m} E(m, 0) I_{n-m}\left(\frac{4 t}{\tau}\right)
$$




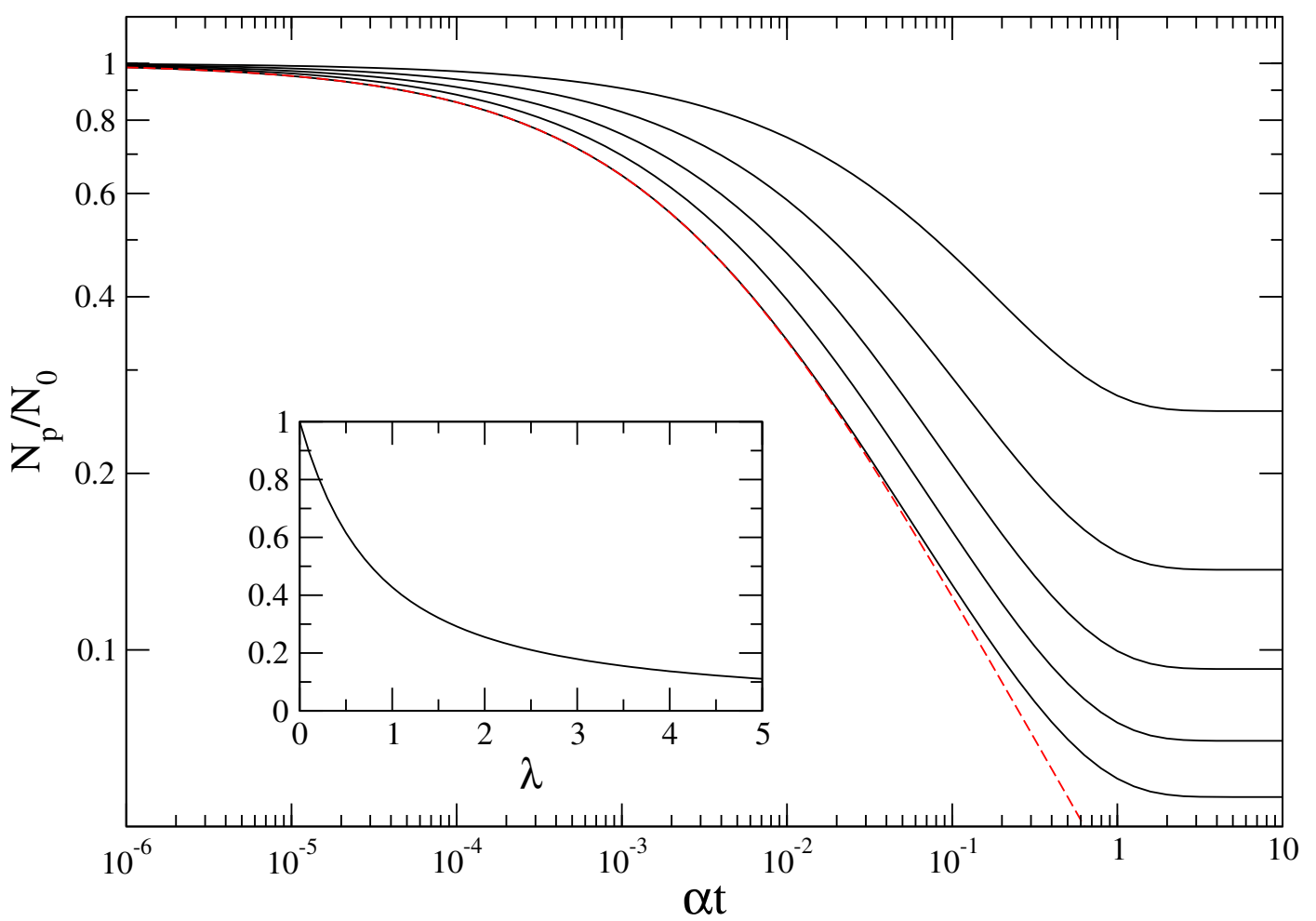

Figure 4. Evaluation of the analytical ratio of the remaining particles $N_{p} / N_{0}$ in the exponential case, equation (19), for several parameters $\lambda=c_{0} \sqrt{\mathscr{D} / \alpha}$, from $\lambda=2$ (top curve) to $\lambda=10$ (bottom curve), and $\alpha=10^{-4}$. The dashed red curve is the comparison with the diffusive case only, see equation (20). Inset: Number of remaining particles as function of $\lambda$.

$=e^{-4 t / \tau}\left[\sum_{m \geq 1} E(m, 0)\left(I_{n-m}\left(\frac{4 t}{\tau}\right)-I_{n+m}\left(\frac{4 t}{\tau}\right)\right)+2 \sum_{m \geq 1} I_{n+m}\left(\frac{4 t}{\tau}\right)+I_{n}\left(\frac{4 t}{\tau}\right)\right]$.

If we start with a lattice filled with one particle per site, $E(m \geq 1,0)=0$, the previous equation is simplified as only the last two terms remain. The particle concentration is given by $c(t)=1-E(1, t)$ and therefore we obtain for the non-expanding lattice case

$$
c(t)=e^{-4 t / \tau}\left[I_{0}\left(\frac{4 t}{\tau}\right)+I_{1}\left(\frac{4 t}{\tau}\right)\right] \simeq \sqrt{\frac{\tau}{2 \pi t}} .
$$

Another way to recover this result in the large time limit and for the same initial conditions is to use the complex integral with a path around the unit circle to obtain the value of $E(n, t)$

$$
\begin{aligned}
& E(n, t)=\oint \frac{d z}{2 i \pi z^{n+1}} G(z, t) \\
& =\oint \frac{d z}{2 i \pi z^{n+1}}\left(1+2 \sum_{m \geq 1} z^{-m}\right) \exp \left(-\frac{4 t}{\tau}+2\left(z+\frac{1}{z}\right) \frac{t}{\tau}\right) .
\end{aligned}
$$

If we set $z=e^{i \theta}$ we obtain

$$
E(n, t)=\int_{-\pi}^{\pi} \frac{d \theta}{2 \pi}\left(e^{-i n \theta}+2 \sum_{m \geq 1} e^{-i(m+n) \theta}\right) \exp \left(-\frac{4 t}{\tau}(1-\cos \theta)\right) .
$$


For $t$ large, we can develop the cosine term for $\theta$ small, and integrate over $\theta$ the resulting Gaussian integral after extending the range of integration to infinity

$$
E(n, t) \simeq \sqrt{\frac{\tau}{8 \pi t}}\left(e^{-n^{2} \tau / 8 t}+2 \sum_{m \geq 1} e^{-(m+n)^{2} \tau / 8 t}\right) .
$$

It is useful to consider the Poisson-Jacobi inversion formula [28]

$$
\sum_{m=-\infty}^{\infty} e^{-\pi t m^{2}-2 \pi m t a}=t^{-1 / 2} e^{\pi a^{2} t}\left(1+2 \sum_{m=1}^{\infty} e^{-\pi m^{2} / t} \cos (2 \pi m a)\right)
$$

to rewrite equation (31) as an asymptotic expansion

$$
\begin{aligned}
E(n, t) & \simeq 1-\sqrt{\frac{\tau}{8 \pi t}}\left(1-e^{-n^{2} \tau / 8 t}+2 \sum_{m=1}^{n} e^{-m^{2} \tau / 8 t}\right) \\
& \simeq 1-n \sqrt{\frac{\tau}{2 \pi t}} .
\end{aligned}
$$

From this long-time approximation it is easy to recover the same asymptotic behavior of the concentration in equation (29).

Let us now apply this technique to the critical value $\beta=1 / 2$ with the same initial conditions. The generating function (26) is equal, after performing the summation over $k$, to

$G(z, t)=F(w r(t)) \frac{\left(1-w^{2}\right) r(t)}{1-w^{2} r^{2}(t)} \exp \left(\frac{4}{\alpha \tau} w^{2} r^{2}(t) \log \left[\frac{\left(1-w^{2}\right) r^{2}(t)}{1-w^{2} r^{2}(t)}\right]\right)$.

The probability $E(n, t)$ is then equal to the contour integral

$$
\begin{aligned}
& E(n, t)=\oint \frac{d z}{2 i \pi z^{n+1}}\left(1+2 \sum_{m \geq 1}\left(\frac{1+w r(t)}{1-w r(t)}\right)^{m}\right) \\
& \times \frac{\left(1-w^{2}\right) r(t)}{1-w^{2} r^{2}(t)} \exp \left(\frac{4}{\alpha \tau} w^{2} r^{2}(t) \log \left[\frac{\left(1-w^{2}\right) r^{2}(t)}{1-w^{2} r^{2}(t)}\right]\right) .
\end{aligned}
$$

As before, for large times, we can use the change of variable $z=e^{i \theta}$ and expand the previous integrand around $\theta=0$, assuming this is the main contribution to the integral. In term of the variable $w$, we have the approximation $w \simeq-i \theta / 2$ and therefore

$$
\begin{aligned}
& E(n, t) \simeq \int_{-\infty}^{\infty} \frac{d \theta}{2 \pi}\left(e^{-i n \theta}+2 \sum_{m \geq 1} e^{-i(m r(t)+n) \theta}\right) \\
& \times \frac{\left(1+\theta^{2} / 4\right) r(t)}{1+\theta^{2} r^{2}(t) / 4} \exp \left(-\frac{\theta^{2} r^{2}(t)}{\alpha \tau} \log \left[\frac{\left(1+\theta^{2} / 4\right) r^{2}(t)}{1+\theta^{2} r^{2}(t) / 4}\right]\right) .
\end{aligned}
$$

In this expression we perform the variable change $r(t) \theta \rightarrow \theta$

$$
\begin{aligned}
E(n, t) & \simeq \int_{-\infty}^{\infty} \frac{d \theta}{2 \pi}\left(e^{-i n \theta / r(t)}+2 \sum_{m \geq 1} e^{-i(m+n / r(t)) \theta}\right) \\
& \times \frac{\left(1+\theta^{2} / 4 r^{2}(t)\right)}{1+\theta^{2} / 4} \exp \left(-\frac{\theta^{2}}{\alpha \tau} \log \left[\frac{r^{2}(t)+\theta^{2} / 4}{1+\theta^{2} / 4}\right]\right) .
\end{aligned}
$$


This integral can be further simplified if we keep the dominant contributions in $1 / r(t)$ and discard the terms in $1 / r^{2}(t)$ :

$$
\begin{aligned}
& E(n, t) \simeq \int_{-\infty}^{\infty} \frac{d \theta}{2 \pi}\left(e^{-i n \theta / r(t)}+2 \sum_{m \geq 1} e^{-i(m+n / r(t)) \theta}\right) \exp \left(-\frac{2 \theta^{2}}{\alpha \tau} \log r(t)\right) \\
& \simeq \sqrt{\frac{\alpha \tau}{8 \pi \log r(t)}}\left[\exp \left(-\frac{\alpha \tau}{8 \log r(t)} \frac{n^{2}}{r^{2}(t)}\right)+2 \sum_{m \geq 1} \exp \left(-\frac{\alpha \tau}{8 \log r(t)}(m+n / r(t))^{2}\right)\right] \\
& \simeq \sqrt{\frac{\alpha \tau}{8 \pi \log r(t)}} \exp \left(-\frac{\alpha \tau}{8 \log r(t)} \frac{n^{2}}{r^{2}(t)}\right)\left[1+2 \sum_{m \geq 1} e^{-\frac{\alpha \tau}{8 \log r(t)} m^{2}}\left(1-\frac{\alpha \tau}{4 \log r(t)} \frac{n}{r(t)} m\right)\right] .
\end{aligned}
$$

We can use in the previous the asymptotic result

$$
\sum_{m \geq 1} m e^{-\pi \epsilon m^{2}} \simeq \epsilon^{-1} \int_{0}^{\infty} d s s e^{-\pi s^{2}} \simeq(2 \pi \epsilon)^{-1}
$$

when $\epsilon$ is small, to obtain the following expansion for the interval probability and concentration

$$
\begin{aligned}
& E(n, t) \simeq 1-\frac{n}{r(t)} \sqrt{\frac{\alpha \tau}{2 \pi \log r(t)}} \\
& r(t) c(t) \simeq \sqrt{\frac{\alpha \tau}{2 \pi \log r(t)}} \propto \log (t)^{-1 / 2} .
\end{aligned}
$$

We recover in this case the asymptotic result in the continuous case given by equation (18).

A more direct and simpler analysis of the discretized diffusion process is to start with a modified form of the transformed equation (10)

$$
\tau \frac{d \mathcal{E}(n, u)}{d u}=2 \Delta \mathcal{E}(n, u)
$$

the solution of which is given formally by equation (28), or

$$
\begin{aligned}
\mathcal{E}(n, u) & =e^{-4 u / \tau}\left[\sum_{m \geq 1} \mathcal{E}(m, 0)\left(I_{n-m}\left(\frac{4 u}{\tau}\right)-I_{n+m}\left(\frac{4 u}{\tau}\right)\right)\right. \\
& \left.+2 \sum_{m \geq 1} I_{n+m}\left(\frac{4 u}{\tau}\right)+I_{n}\left(\frac{4 u}{\tau}\right)\right]
\end{aligned}
$$

with $\mathcal{E}(m, 0)=E(m, 0)$. Then, after substituting the original time and space variables $u \rightarrow u(t)$ and $n \rightarrow n r^{-1}(t)$, we obtain

$$
\begin{aligned}
E(n, t) & =e^{-4 u(t) / \tau}\left[\sum_{m \geq 1} E(m, 0)\left(I_{n / r(t)-m}\left(\frac{4 u(t)}{\tau}\right)-I_{n / r(t)+m}\left(\frac{4 u(t)}{\tau}\right)\right)\right. \\
& \left.+2 \sum_{m \geq 1} I_{n / r(t)+m}\left(\frac{4 u(t)}{\tau}\right)+I_{n / r(t)}\left(\frac{4 u(t)}{\tau}\right)\right] .
\end{aligned}
$$


From this result, the concentration is given, with a lattice initially filled with particles as initial condition, by the expression

$$
c(t)=1-e^{-4 u(t) / \tau}\left[2 \sum_{m \geq 1} I_{1 / r(t)+m}\left(\frac{4 u(t)}{\tau}\right)+I_{1 / r(t)}\left(\frac{4 u(t)}{\tau}\right)\right] .
$$

The number of remaining particles is given by $N_{p}(t) / N_{0}=r(t) c(t)$, with $N_{0}$ the number of initial lattice sites which is equal to the initial number of particles. The comparison with the continuous case, equation (18), is displayed in the inset of figure 2 for $\beta=0.2$. We have set $\sqrt{\mathscr{D}} c_{0}=\sqrt{a^{2} / \tau} N_{0} /\left(N_{0} a\right)=\sqrt{1 / \tau}$ and expressed the other parameters with $\alpha \tau=1$. As expected, the large time limit of both cases are equivalent.

\section{Analogy with geometrical expanding structures}

In this section we would like to discuss the applicability of the previous results to the case of growth models on particular geometrical structures in two dimensions. We can indeed interpret the time dilatation process as the increase of the available space a random particle is allowed to move into at every time step, due only to geometrical constraints. As an example, let us consider the properties of a directed growth structure on a disk domain, see references $[9,29,11]$, such as depicted in figure $5(\mathrm{a})$. We can see that the trajectory tends to develop on a circle of growing radius and therefore in a larger space as time increases. The equation of diffusion is therefore modified by the expansion of the space. Following the same idea as in reference [9], we note $\rho_{k}=\rho_{0}+k d \rho$ the radius of the circle where the particle or arm is located at step $k$ with increments $d \rho$. Originally the particle is located at radius $\rho_{0}$, and we consider a Brownian motion such as equation (2), but with variable $y_{k} \in\left[0,2 \pi \rho_{k}\right]$ measuring the displacement length of the particle on the corresponding circle

$$
y_{k}=\frac{r_{k}}{r_{k-1}}\left(y_{k-1}+\xi_{k}\right) \text {. }
$$

The growth factor $r_{k}=\left(1+\beta^{-1} k d \rho / \rho_{0}\right)^{\beta}$ is needed to take into account the expansion of the radius domain at every step. For a disk geometry [9], such as considered in figure $5(\mathrm{a}), \beta$ is unity, but here we generalize this model to any geometry characterized by the scaling exponent $\beta$, as in equation (1), characterizing the expansion rate.

The mapping onto a linear or strip domain of fixed width $L_{0}=2 \pi \rho_{0}$, see figure $5(\mathrm{~b})$, is made possible by considering the transformed process $x_{k}=\left(\rho_{0} / \rho_{k}\right) y_{k} \in[0, L]$. The new variables $x_{k}$ satisfy a modified Brownian motion

$$
x_{k}=x_{k-1}+\frac{\xi_{k}}{r_{k-1}}, x_{k}=\sum_{i=1}^{k} \frac{\xi_{i}}{r_{i-1}}+x_{0} .
$$

This equation is similar to a classical free Brownian trajectory except that the noise is multiplied by a non-constant scaling factor which modifies the properties of the dynamics. However it is possible to map the radius $\rho_{k}$ into an effective height $h_{k}$, see figure $5(\mathrm{~b})$, with the condition $h_{0}=0$, by considering the variance of the $x_{k}$, and using the same noise definition as in equation $(4):\left\langle\xi_{i} \xi_{j}\right\rangle=2 a^{2} \delta_{i, j}$ 


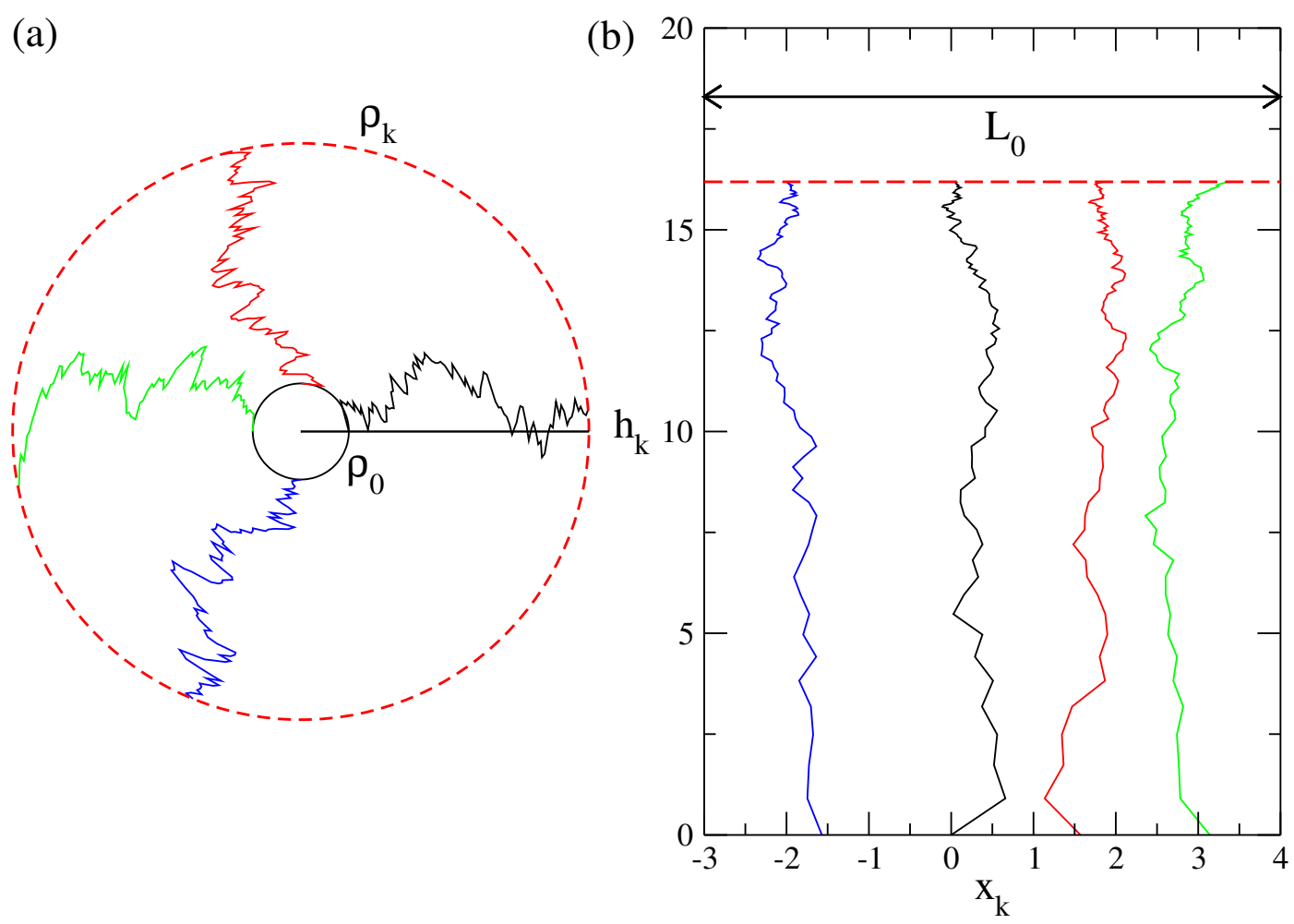

Figure 5. Brownian motion on a circle (a) and mapping onto a linear domain (b), see references $[9,29]$ figure $1 . \rho_{k}$ is the radius of the arm structure at time step $k$, and the radius maps onto an effective height $h_{k}$ in the linear domain of fixed width $L_{0}=2 \pi \rho_{0}$.

$$
h_{k}=\left\langle\left(x_{k}-x_{0}\right)^{2}\right\rangle=2 a^{2} \sum_{i=1}^{k} \frac{1}{r_{i-1}^{2}} .
$$

In the continuous limit $k d \rho \rightarrow \rho-\rho_{0}$ and $a^{2} / d \rho \rightarrow \mathscr{D}$ we obtain

$$
\begin{aligned}
& h(\rho)=2 \mathscr{D} \frac{\beta \rho_{0}}{1-2 \beta}\left[\left(1+\beta^{-1}\left(\rho-\rho_{0}\right) / \rho_{0}\right)^{1-2 \beta}-1\right], \beta \neq \frac{1}{2} \\
& h(\rho)=\mathscr{D} \rho_{0} \log \left[\left(\rho+\rho_{0}\right) / 2 \rho_{0}\right], \beta=\frac{1}{2}
\end{aligned}
$$

which is equivalent to the effective or comoving diffusive length given by equation (5) with $\alpha=1 / \rho_{0}$. The role played by the time variable is replaced by the radius difference $\rho-\rho_{0}$ of the growing structure and the mapping in figure 5 shows that the projected height $h(\rho)$ takes a finite value when $\beta>1 / 2$, in the limit $\rho \rightarrow \infty$. The corresponding relation between the exponent $\gamma$ in reference [29] and $\beta=(2 \gamma)^{-1}$ is only valid for the growth structure on the disk, figure 5(a), and for a Brownian noise $\gamma=1 / 2$ or $\beta=1$. In reference [29], the authors consider instead the growth of structures on a disk with different kinds of noise (Levy flights, fractional Brownian motion, see also [30, 31]) with $\gamma$ related to the fluctuation scaling $\delta y_{k} \sim\left(\delta \rho_{k}\right)^{\gamma}$ of the arm structures of figure $5(\mathrm{a})$, and therefore the previous scaling relation between $\beta$ and $\gamma$ is not applicable in the general case. Instead, in the present study, $\beta$ is a geometrical exponent related to the domain curvature while the noise is purely Gaussian. 
For $\beta=1 / 2$, equation (48) is the usual conformal mapping from the outside circle $\rho \geq \rho_{0}$ to the strip of width $L_{0}$ and $h(\rho) \geq 0$. Also, for $\rho$ close to $\rho_{0}$ we have the local linear relation $h(\rho) \simeq 2 \mathscr{D}\left(\rho-\rho_{0}\right)$ between the two domains at small times. Finally, it is worth noticing that $h_{k}$ is the time change such that if $B_{k}=\sum_{i=1}^{k} \xi_{i}$ is a Wiener process, $B_{h_{k}}=\sum_{i=1}^{k} \xi_{i} / r_{i-1}$ is also a Wiener process $\left\langle B_{h_{k}} B_{h_{l}}\right\rangle=\min \left(h_{k}, h_{l}\right)$ with respect to the new variable $h_{k}$.

\section{Modelization in term of fractional diffusion}

We have seen that a space dilatation with algebraic or geometrical exponent $\beta<1 / 2$ leads to the one-particle absorbing state with a power law decay proportional to $t^{\beta-1 / 2}$ representing a subdiffusive dynamics. We can try to modelize this abnormal diffusion using the formalism of fractional dynamics [21, 29, 32]. This idea is to incorporate phenomenologically the effect of the expansion or dilution into a single fractional operator acting on the diffusion process itself, modifying the effective exponent of the diffusion. We would like to see in details how the diffusion length is characterized and if the fractional formalism describes accurately the previous results. The time evolution of the empty intervals, equation (9), can instead be described by considering a fractional diffusion equation for the IPDF [21]

$$
\begin{aligned}
\frac{\partial E(x, t)}{\partial t} & ={ }_{0} D_{t}^{1-2 \nu} 2 K_{\nu} \frac{\partial^{2} E(x, t)}{\partial x^{2}} \\
& =\frac{2 K_{\nu}}{\Gamma(2 \nu)} \frac{\partial}{\partial t} \int_{0}^{t} \frac{d t^{\prime}}{\left(t-t^{\prime}\right)^{1-2 \nu}} \frac{\partial^{2} E\left(x, t^{\prime}\right)}{\partial x^{2}}
\end{aligned}
$$

where the Grünwald-Letnikov operator ${ }_{0} D_{t}^{1-2 \nu}$ (see [33] for the technical details) ensures that the mean squared displacement of a particle, or fractional diffusive length, is subdiffusive: $\ell_{f}^{2}(t) \simeq 2 K_{\nu} t^{2 \nu} / \Gamma(1+2 \nu)$ with $0<\nu \leq 1 / 2$. We assume that equation (49) describes correctly the physics of the model for the IPDF given by equation (9), and that $\ell_{f}(t)$ is associated to $\ell(t)$, equation (5). Exponents $\nu$ and $\beta$ are therefore related by the identity $\nu=1 / 2-\beta$, and constant $K_{\nu}$ can be identified here with the diffusion coefficient. We find in particular when $t \gg 1$

$$
K_{\nu} \simeq \frac{\mathscr{D}(1-2 \nu)}{4 \alpha \nu}\left(\frac{2 \alpha}{1-2 \nu}\right)^{2 \nu} \Gamma(1+2 \nu) \text {. }
$$

It is known that the solution of equation (49) gives the concentration expression in term of a Mittag-Leffler function[21] which has the following asymptotic expansion [34]

$$
\frac{N_{p}(t)}{N_{0}}=E_{\nu}\left(-c_{0} \sqrt{2 K_{\nu}} t^{\nu}\right) \simeq \frac{\left(2 K_{\nu}\right)^{-1 / 2}}{c_{0} \Gamma(1-\nu)} t^{-\nu}
$$

In our model, equation (18), we have instead asymptotically, in the regime $\beta<1 / 2$

$$
\frac{N_{p}(t)}{N_{0}} \simeq \frac{\Gamma(1+2 \nu)^{1 / 2}}{c_{0}\left(2 \pi K_{\nu}\right)^{1 / 2}} t^{-\nu} .
$$

The two asymptotic behaviors give the same time dependence, but differ by the two numerical coefficients $\Gamma(1-\nu)^{-1}$ and $[\Gamma(1+2 \nu) / \pi]^{1 / 2}$, depending on the equation 
considered to model the diffusion. These two coefficients coincide only for the normal Brownian diffusion $\left(\nu=1 / 2\right.$ or $\beta=0$ where $\left.K_{1 / 2}=\mathscr{D}\right)$. We should also notice that the particular case $E_{1 / 2}(-x)=e^{x^{2}} \operatorname{erfc}(x)$ corresponds to the IPDF found in equation (18). However equations (49) and (51) do not describe the localized regime for which $\beta \geq 1 / 2$ (in this case $\nu$ would be negative). In the limit $\beta=1 / 2$ or $\nu \rightarrow 0$, we can however recover the logarithmic behavior of the effective length as $K_{\nu} \simeq \mathscr{D} /(4 \alpha \nu)$ and $\ell_{f}^{2}(t) \simeq 2 K_{\nu} e^{2 \nu \log (t)} \simeq 4 \nu K_{\nu} \log (t) \simeq(\mathscr{D} / \alpha) \log (t)$.

Let us first discuss the subdiffusive regime which can be studied in the framework of CTRW models [35, 20, 36, 37] related to fractional diffusion, where time and space steps are distributed from a probability function. Let consider a random process where time steps, or waiting times $\tau_{i}$, are taken from the following distribution

$$
\varphi\left(\tau_{i}\right)=\frac{\tau_{c}^{2 \nu}}{\Gamma(2 \nu)} \frac{e^{-\tau_{c} / \tau_{i}}}{\tau_{i}^{1+2 \nu}}
$$

where $\tau_{c}$ is some short time cutoff that is necessary to normalize the distribution and $\nu>0$ an arbitrary exponent not necessary equal to $1 / 2-\beta$. The distribution has a finite mean $\tau=\left\langle\tau_{i}\right\rangle$, only if $\nu>1 / 2: \tau=\tau_{c} /(2 \nu-1)$. We should notice that a different distribution such as $\varphi\left(\tau_{i}\right)=2 \nu \tau_{c}^{2 \nu} /\left(\tau_{c}+\tau_{i}\right)^{1+2 \nu}$ would give similar results. We also assume that space steps $a_{i}$ are taken from a Gaussian distribution $\lambda\left(a_{i}\right)=e^{-a_{i}^{2} / 4 a^{2}} / \sqrt{4 \pi a^{2}}$ [37]. Its Fourier transform is given by $\tilde{\lambda}(k)=e^{-k^{2} a^{2}} \simeq 1-a^{2} k^{2}$. We consider in this context the Green function $W(x, t)$, which is the probability to find a particle at location $x$ at time $t$. For small momenta, the Fourier-Laplace transform $\bar{W}(k, s)$ of $W(x, t)$ satisfies a Montroll-Weiss equation [38, 39, 37]

$$
s \bar{W}(k, s)=1-\hat{\varphi}(s)+s \hat{\varphi}(s) \tilde{\lambda}(k) \bar{W}(k, s)
$$

which can be rewritten in the present case as

$$
s \bar{W}(k, s)=1-a^{2} k^{2} \frac{s \hat{\varphi}(s)}{1-\hat{\varphi}(s)} \bar{W}(k, s)
$$

where

$$
\hat{\varphi}(s)=\frac{2\left(\tau_{c} s\right)^{\nu}}{\Gamma(2 \nu)} K_{2 \nu}\left(2 \sqrt{\tau_{c} s}\right) .
$$

In the long time limit or for small $s$, we find the following expansion

$$
\begin{aligned}
& \hat{\varphi}(s) \simeq 1-\frac{\Gamma(1-2 \nu)}{2 \nu \Gamma(2 \nu)}\left(\tau_{c} s\right)^{2 \nu}, \nu<\frac{1}{2}, \\
& \hat{\varphi}(s) \simeq 1-\left(\tau_{c} s\right) \log \left(\frac{e^{1-2 \gamma}}{\tau_{c} s}\right), \nu=1 / 2, \\
& \hat{\varphi}(s) \simeq 1-\frac{\tau_{c} s}{2 \nu-1}, \nu>\frac{1}{2} .
\end{aligned}
$$

Using the first term of the previous equation when $\nu<1 / 2$, we have

$$
s \bar{W}(k, s) \simeq 1-\frac{a^{2} k^{2}}{\tau_{c}^{2 \nu}} \frac{2 \nu \Gamma(2 \nu)}{\Gamma(1-2 \nu)} s^{1-2 \nu} \bar{W}(k, s)
$$


from which we can inverse the Laplace and Fourier transforms, and obtain the fractional diffusion equation for the subdiffusive regime $\nu<1 / 2$

$$
\begin{aligned}
& \frac{\partial W(x, t)}{\partial t} \simeq \frac{K_{\nu}}{\Gamma(2 \nu)} \frac{\partial}{\partial t} \int_{0}^{t} \frac{d t^{\prime}}{\left(t-t^{\prime}\right)^{1-2 \nu}} \frac{\partial^{2} W\left(x, t^{\prime}\right)}{\partial x^{2}}, \\
& K_{\nu}=\frac{2 \nu a^{2} \Gamma(2 \nu)}{\tau_{c}^{2 \nu} \Gamma(1-2 \nu)} .
\end{aligned}
$$

We have identified a diffusive factor $K_{\nu}$, as in equation (49), but without the factor 2 which is normally found for the IPDF. For the marginal case $\nu=1 / 2$ we find

$$
\begin{aligned}
& \frac{\partial W(x, t)}{\partial t} \simeq \frac{\partial}{\partial t} \int_{0}^{t} d t^{\prime} \int_{0}^{1} \frac{K_{\nu} d u}{\Gamma(1-u)}\left(\frac{e^{1-2 \gamma}}{t-t^{\prime}}\right)^{u} \frac{\partial^{2} W\left(x, t^{\prime}\right)}{\partial x^{2}}, \\
& K_{\nu}=\frac{a^{2}}{\tau_{c}} .
\end{aligned}
$$

This expression comes from the identity when $s$ is small

$$
\mathcal{L}^{-1}\left(\frac{s-1}{\log s}\right)=\partial_{t} \int_{0}^{1} d u \frac{t^{-u}}{\Gamma(1-u)} \simeq \mathcal{L}^{-1}\left(-\frac{1}{\log s}\right)
$$

which is correct in the long time limit. In the diffusive case $\nu>1 / 2$, we recover the usual equation of diffusion

$$
\begin{aligned}
& \frac{\partial W(x, t)}{\partial t}=K_{\nu} \frac{\partial^{2} W(x, t)}{\partial x^{2}}, \\
& K_{\nu} \simeq \frac{a^{2}(2 \nu-1)}{\tau_{c}}=\frac{a^{2}}{\tau}=\mathscr{D} .
\end{aligned}
$$

The fractional diffusive length $\ell_{f}(t)$ can be evaluated using the definition in term of the Laplace transform

$$
\mathcal{L}\left(\ell_{f}^{2}(t)\right)=-\left.\frac{\partial^{2} \bar{W}(k, s)}{\partial k^{2}}\right|_{k=0}=\frac{2 a^{2} \hat{\varphi}(s)}{s(1-\hat{\varphi}(s))} .
$$

Using the three expansions of equation (57), we obtain the following results in the long time limit after taking the inverse Laplace transforms

$$
\begin{aligned}
& \ell_{f}^{2}(t) \simeq \frac{2 a^{2}}{\tau_{c}^{2 \nu} \Gamma(1-2 \nu)} t^{2 \nu}, \nu<1 / 2, \\
& \ell_{f}^{2}(t) \simeq \frac{2 a^{2}}{\tau_{c}} \frac{t}{\log \left(e^{1-2 \gamma} t / \tau_{c}\right)}, \nu=1 / 2 \\
& \ell_{f}^{2}(t) \simeq \frac{2 a^{2}(2 \nu-1)}{\tau_{c}} t=2 \mathscr{D} t, \nu>1 / 2 .
\end{aligned}
$$

In the marginal case, $\nu=1 / 2$, we have used the identities

$$
\begin{aligned}
& \mathcal{L}^{-1}\left(\frac{1-s^{-2}}{\log s}\right)=\int_{0}^{2} d u \frac{t^{1-u}}{\Gamma(2-u)} \simeq \mathcal{L}^{-1}\left(-\frac{1}{s^{2} \log s}\right), \\
& \int_{0}^{2} d u \frac{t^{1-u}}{\Gamma(2-u)} \simeq \frac{t}{\log (t)}, \text { for } t \gg 1 .
\end{aligned}
$$

The fractional diffusion dynamics characterized by equations (64) represents therefore a subdiffusive process for which $\ell_{f}^{2}(t)$ cannot exceed $t$, as in equation (5). When $\nu>1 / 2$, 
the time distribution for the $\tau_{i}$, equation (53), has a finite mean which implies that the diffusive process is Brownian. In the opposite case, when $\nu<0$, we can argue that the waiting times are infinite and therefore the particles appear to be localized.

From the point of view of Lévy process, we can also estimate the fractional diffusive length using simple scaling relations. If $N_{t}$ is the number of the particle steps, then $\ell_{f}^{2}(t) \sim a^{2} N_{t}$. The time duration for performing $N_{t}$ independent steps scales like $t=\tau_{1}+\cdots+\tau_{N_{t}} \sim N_{t}^{1 / 2 \nu}$ [40] according to the distribution properties of equation (53) which has a divergent mean value when $\nu<1 / 2$, and proportional to $N_{t}$ when $\nu>1 / 2$. We therefore recover the fact that $\ell_{f}^{2}(t) \sim t^{2 \nu}$ and $\ell_{f}^{2}(t) \sim t$ respectively.

The fractional case describing subdiffusive processes can be compared to the effective diffusive length $\ell(t)$ of the expansion model obtained in equation (5). Asymptotically we deduce that

$$
\begin{aligned}
\ell^{2}(t) & \sim t^{1-2 \beta}, \beta<1 / 2, \\
\ell^{2}(t) & \sim \log (t), \beta=1 / 2, \\
\ell^{2}(t) & \sim 1, \beta>1 / 2 .
\end{aligned}
$$

Only the first case $\beta<1 / 2$ could be identified to the fractional diffusion, equation (64), when $\nu<1 / 2$ by substituting $\nu=1 / 2-\beta$. However, it is more interesting to consider the case where $\nu=\beta$ in equation (64). Then the product $\ell^{2}(t) \ell_{f}^{2}(t)$ is always proportional to $t$ for any value of $\beta$, i.e. proportional to the squared of the classical Brownian diffusive length. This reflects the duality between the diffusion on an expanding space and the fractional model of waiting times distributed with an algebraic law.

If we consider instead the physical length $\ell_{\text {phys }}(t)=r(t) \ell(t)$ rather than the comoving length $\ell(t)$, we have the following long-time behavior

$$
\begin{aligned}
& \ell_{\text {phys }}^{2}(t) \sim t, \beta<1 / 2, \\
& \ell_{\text {phys }}^{2}(t) \sim t \log (t), \beta=1 / 2, \\
& \ell_{\text {phys }}^{2}(t) \sim t^{2 \beta}, \beta>1 / 2 .
\end{aligned}
$$

It is clear that equation (67) represents a superdiffusive process, in opposition to equation (64), and with the physical length $\ell_{\text {phys }}(t)$ scaling like the expansion rate $r(t)$ when $\beta>1 / 2$.

Instead of considering a distribution of waiting times, let assume that the time steps are constant and equal to $\tau$, and that the space increments $a_{i}$ s are taken from the distribution $\lambda\left(a_{i}\right)$ whose Fourier transform is equal to

$$
\lambda\left(a_{i}\right)=\frac{(1+\nu) \sin \left(\frac{\pi}{2(1+\nu)}\right)}{\pi a} \frac{1}{1+\left|a_{i} / a\right|^{2+2 \nu}}
$$

where $\nu>0$ is the exponent of the algebraic decay and $a$ a typical length. For $\nu \leq 1 / 2$ the distribution has an infinite variance. Its Fourier transform is given by

$$
\tilde{\lambda}(k)=\frac{2(1+\nu) \sin \left(\frac{\pi}{2(1+\nu)}\right)}{\pi} \int_{0}^{\infty} d u \frac{\cos (k a u)}{1+u^{2+2 \nu}} .
$$


The behavior of $\tilde{\lambda}(k)$ for small momenta depends on the value of $\nu$. We can rewrite the previous expression in the case $\nu>1 / 2$ as

$$
\begin{aligned}
\tilde{\lambda}(k) & =\frac{2(1+\nu) \sin \left(\frac{\pi}{2(1+\nu)}\right)}{\pi}\left(\int_{0}^{\infty} \frac{1}{1+u^{2+2 \nu}} d u+\int_{0}^{\infty} \frac{\cos (k a u)-1}{1+u^{2+2 \nu}} d u\right) \\
& \simeq 1-\frac{(1+\nu) \sin \left(\frac{\pi}{2(1+\nu)}\right)}{\pi}(k a)^{2} \int_{0}^{\infty} \frac{u^{2}}{1+u^{2+2 \nu}} d u \\
& \simeq 1-\left(a_{\nu} k\right)^{2}, \nu>1 / 2
\end{aligned}
$$

with $a_{\nu}^{2}=\left(4 \cos ^{2}[\pi /(2+2 \nu)]-1\right) a^{2} / 2$. The diffusion is Brownian with a length scale proportional to $t^{1 / 2}$. In the case $\nu<1 / 2$, the integral in the second line of equation (70) is divergent. We can write instead

$$
\begin{aligned}
\tilde{\lambda}(k) & \simeq 1+\frac{2(1+\nu) \sin \left(\frac{\pi}{2(1+\nu)}\right)}{\pi}|k a|^{-1} \int_{0}^{\infty} \frac{(\cos (u)-1) d u}{1+(u /|k a|)^{2+2 \nu}} \\
& \simeq 1-\frac{2(1+\nu) \sin \left(\frac{\pi}{2(1+\nu)}\right)}{\pi}|k a|^{1+2 \nu} \int_{0}^{\infty} \frac{(1-\cos (u)) d u}{u^{2+2 \nu}} \\
& =1-\left(a_{\nu} k\right)^{1+2 \nu}, \nu<1 / 2 .
\end{aligned}
$$

The last integral converges around 0 and at infinity when $\nu<1 / 2$. Using $\hat{\varphi}(s)=e^{-\tau s} \simeq$ $1-\tau s$ we can solve the Montroll-Weiss equation (54) and find

$$
\bar{W}(k, s) \simeq \frac{1}{s+\tau^{-1}\left|a_{\nu} k\right|^{1+2 \nu}}, \nu<1 / 2 .
$$

The Laplace inverse gives simply

$$
\mathcal{L}^{-1}(\bar{W}(k, s))=\exp \left(-\tau^{-1}\left|a_{\nu} k\right|^{2 \nu+1} t\right)
$$

and the Green function is expressed as

$$
\begin{aligned}
W(x, t) & =\left(\frac{\tau}{t}\right)^{1 /(2 \nu+1)} \int \frac{d u}{2 \pi a_{\nu}} e^{-i u(\tau / t)^{1 /(2 \nu+1)} x / a_{\nu}-|u|^{2 \nu+1}} \\
& =a_{\nu}^{-1}\left(\frac{\tau}{t}\right)^{1 /(2 \nu+1)} F_{\nu}\left(\left(\frac{\tau}{t}\right)^{1 /(2 \nu+1)} x / a_{\nu}\right) .
\end{aligned}
$$

The function $F_{\nu}$ is a Levy distribution [39] with exponent $2 \nu+1$. When $\nu=1 / 2$ we recover the Gaussian density $F_{1 / 2}(y)=e^{-y^{2} / 4} / 2 \sqrt{\pi}$ and the fact that the diffusive length scales like $t^{1 / 2}$. For $\nu<1 / 2$ the variance of the function $\lambda$ is infinite and the diffusive length is not defined. However the scaling of the function (74) would formally gives a length scale growing like $t^{1 /(2 \nu+1)}$, with an exponent larger than $1 / 2$ and less than unity.

This superdiffusive scaling can be achieved using a truncated Levy flight process by discarding the large deviations beyond a cutoff length [41]. Although the Gaussian behavior should be recovered in the large time limit, we expect to see a superdiffusive regime for some time interval. The diffusive length squared should indeed scale like $\sum_{i=1}^{N_{t}} a_{i}^{2} \sim N_{t}^{2 /(2 \nu+1)} \sim t^{2 /(2 \nu+1)}$ for $N_{t}$ time steps of duration $\tau$. 


\section{Conclusion}

We have investigated in this paper the limiting effect of an inflating space on the reaction process of coagulation-diffusion for Brownian particles, in particular we have obtained exact solutions when the space is discrete for a general initial distribution of particles and for a given expansion law characterized by the algebraic exponent $\beta$, using generating functions. The results of the continuous model are recovered in the large time limit. This model possesses two regimes which can be understood in the steady state limit as a second order phase transition between an absorbing state $N_{p}=0$ and a localized state $N_{p} \neq 0$, with a slowing down phenomenon at the critical point $\beta=1 / 2, \beta$ being identified with an inverse temperature.

An analogy is made with growth systems constrained by radial geometries and for which the noise is Gaussian. We extended the problem to expanding geometries which generalizes the radial case.

We finally compare these results with fractional models of waiting time distributions, and found a duality relation between the effective diffusive lengths. More precisely, for the same class of exponent, the product of the effective length $\ell(t)$ in the model of space expansion with the diffusive length $\ell_{f}(t)$ given by the fractional representation of the diffusion operator is always proportional to $t^{1 / 2}$, which is the effective length of the pure Brownian motion, even at criticality.

\section{Acknowledgments}

This work was supported by Brain Pool Program through the National Research Foundation of Korea (Grant No. NRF-2018H1D3A2065321) and in part through the Basic Science Research Program (Grants No. 2016R1D1A1A09917318 and 2019R1F1A1046285).

\section{References}

[1] D. ben Avraham and S. Havlin. Diffusion and reactions in fractals and disordered systems. Cambridge University Press, 2000.

[2] M. Henkel, H. Hinrichsen, and S. Lübeck. Non-equilibrium phase transitions: absorbing phase transitions, volume 1. Springer, Heidelberg, 2008.

[3] G. Ódor. Universality in non-equilibrium lattice systems. World Scientific, Singapore, 2008.

[4] Ron Kroon, Hilde Fleurent, and Rudolf Sprik. Diffusion-limited exciton fusion reaction in onedimensional tetramethylammonium manganese trichloride (tmmc). Phys. Rev. E, 47:2462-2472, 1993.

[5] R. M. Russo, E. J. Mele, C. L. Kane, I. V. Rubtsov, M. J. Therien, and D. E. Luzzi. Onedimensional diffusion-limited relaxation of photoexcitations in suspensions of single-walled carbon nanotubes. Phys. Rev. B, 74:R041405, 2006.

[6] J. L. Spouge. Exact solutions for a diffusion-reaction process in one dimension. Phys. Rev. Lett., 60:871, 1988. erratum 60 (1988) 1885.

[7] Charles R. Doering and Daniel ben Avraham. Diffusion-limited coagulation in the presence of particle input: Exact results in one dimension. Phys. Rev. Lett., 62:2563-2566, 1989. 
[8] Haye Hinrichsen, Vladimir Rittenberg, and Horatin Simon. Universality properties of the stationary states in the one-dimensional coagulation-diffusion model with external particle input. J. Stat. Phys., 86(5-6):1203-1235, 1997.

[9] Adnan Ali, Robin C. Ball, Stefan Grosskinsky, and Ellák Somfai. Scale-invariant growth processes in expanding space. Phys. Rev. E, 87:020102, 2013.

[10] S. B. Yuste, E. Abad, and C. Escudero. Diffusion in an expanding medium: Fokker-Planck equation, Green's function, and first-passage properties. Phys. Rev. E, 94(3):032118, 2016.

[11] F. Le Vot, C. Escudero, E. Abad, and S. B. Yuste. Encounter-controlled coalescence and annihilation on a one-dimensional growing domain. Phys. Rev. E, 98(3):032137, 2018.

[12] Ashok Bhakta and Eli Ruckenstein. Decay of standing foams: drainage, coalescence and collapse. Adv. Colloid. Interfac., 70:1-124, 2003.

[13] Marco Mancini. Structure and Evolution of Soap-Like Foams. PhD thesis, Université CergyPontoise, 2005.

[14] V. Berezinsky and A. Z. Gazizov. Diffusion of Cosmic Rays in the Expanding Universe. I. Astrophys. J., 643(1):8-13, 2006.

[15] Adnan Ali, Ellák Somfai, and Stefan Grosskinsky. Reproduction-time statistics and segregation patterns in growing populations. Phys. Rev. E, 85(2):021923, 2012.

[16] Christian A. Yates. Discrete and continuous models for tissue growth and shrinkage. J. Theor. Biol., 350:37-48, 2014.

[17] Matthew J. Simpson. Exact solutions of linear reaction-diffusion processes on a uniformly growing domain: Criteria for successful colonization. PLoS ONE, 10(2):e0117949, 2015.

[18] Oskar Hallatschek, Pascal Hersen, Sharad Ramanathan, and David R Nelson. Genetic drift at expanding frontiers promotes gene segregation. Proc. Natl. Acad. Sci., 104(50):19926-19930, 2007.

[19] Carey D. Nadell, Knut Drescher, and Kevin R. Foster. Spatial structure, cooperation and competition in biofilms. Nat. Rev. Microbiol., 14(9):589-600, 2016.

[20] Ralf Metzler and Joseph Klafter. The random walk's guide to anomalous diffusion: A fractional dynamics approach. Phys. Rep., 339(1):1-77, 2000.

[21] S. B. Yuste and Katja Lindenberg. Subdiffusion-limited A + A reactions. Phys. Rev. Lett., 87(11):118301, 2001.

[22] Jae Hyung Jeon, Aleksei V. Chechkin, and Ralf Metzler. Scaled Brownian motion: A paradoxical process with a time dependent diffusivity for the description of anomalous diffusion. Phys. Chem. Chem. Phys., 16(30):15811-15817, 2014.

[23] Daniel ben Avraham. Complete exact solution of diffusion-limited coalescence, $A+A \rightarrow A$. Phys. Rev. Lett., 81:4756-4759, 1998.

[24] A P Prudnikov, Yu A Brychkov, and O I Marichev. Integrals and Series, volume 5 Inverse Laplace Transforms. Gordon and Breach Science Publishers, New York, 1992. page 52.

[25] X. Durang, J.-Y. Fortin, D. Del Biondo, M. Henkel, and J. Richert. Exact correlations in the one-dimensional coagulation-diffusion process investigated by the empty-interval method. $J$. Stat. Mech., page P04002, 2010.

[26] R S Pathak and H D Chaubey. Poisson-Jacobi transforms. Proc. Indian Acad. Sci., 82(5):191-210, 1975.

[27] John L. Spouge. Exact solutions for a diffusion-reaction process in one dimension. Phys. Rev. Lett., 60(10):871-874, 1988.

[28] E.T. Whittaker and G.N. Watson. A Course of Modern Analysis. A Course of Modern Analysis: An Introduction to the General Theory of Infinite Processes and of Analytic Functions, with an Account of the Principal Transcendental Functions. Cambridge University Press, 1996.

[29] Adnan Ali, Robin C. Ball, S. Grosskinsky, and E. Somfai. Interacting particle systems in timedependent geometries. J. Stat. Mech. Theory Exp., 2013(9):P09006, 2013.

[30] Bernt Øksendal. Stochastic Differential Equations: An Introduction with Applications. SpringerVerlag, 5 edition, 2000. 
[31] Marjorie G. Hahn, Kei Kobayashi, and Sabir Umarov. Fokker-Planck-Kolmogorov equations associated with time-changed fractional Brownian motion. Proc. Am. Math. Soc., 139(02):691691, 2011.

[32] C. N. Angstmann, B. I. Henry, and A. V. McGann. Generalized fractional diffusion equations for subdiffusion in arbitrarily growing domains. Phys. Rev. E, 96(4):042153, 2017.

[33] Rudolf Hilfer. Applications of Fractional Calculus in Physics. World Scientific Publishing Company, Singapore, 2000. Page 9, equation 1.20.

[34] Francesco Mainardi. On some properties of the Mittag-Leffler function $E_{\alpha}\left(-t^{\alpha}\right)$, completely monotone for $t>0$ with $0<\alpha<1$. Discret. Contin. Dyn. Syst.-Ser. B, 19(7):2267-2278, 2014.

[35] Bruce J. West, Paolo Grigolini, Ralf Metzler, and Theo F. Nonnenmacher. Fractional diffusion and Lévy stable processes. Phys. Rev. E, 55(1):99-106, 1997.

[36] E. Barkai, R. Metzler, and J. Klafter. From continuous time random walks to the fractional Fokker-Planck equation. Phys. Rev. E, 61(1):132-138, 2000.

[37] F. Le Vot and S. B. Yuste. Continuous-time random walks and Fokker-Planck equation in expanding media. Phys. Rev. E, 98(4):42117, 2018.

[38] Elliott W. Montroll and Harvey Scher. Random walks on lattices. IV. Continuous-time walks and influence of absorbing boundaries. J. Stat. Phys., 9(2):101-135, 1973.

[39] Ralf Metzler, Aleksei V. Chechkin, and Joseph Klafter. Levy statistics and anomalous transport: Levy flights and subdiffusion. In Robert A. Meyers, editor, Encyclopedia of Complexity and Systems Science, pages 5218-5239. Springer New York, New York, 2009.

[40] Valentin V. Petrov. Limit theorems of probability theory: sequences of independent random variables. Oxford studies in probability 4. Clarendon Press; Oxford University Press, 1995.

[41] Rosario N. Mantegna and H. Eugene Stanley. Stochastic Process with Ultraslow Convergence to a Gaussian: The Truncated Lévy Flight. Phys. Rev. Lett., 73(22):2946, 1994. 